\title{
Isolated Cardiac Hydatid Cyst Causing Complete Heart Block
}

\author{
Ismahane Lahmidi $^{1}$, Mohamed Boutaybi ${ }^{1}$, Jamal El Ouazzani ${ }^{1}$, Noha Elouafi ${ }^{1}$, Zakaria Bazid ${ }^{1}$ \\ 1. Cardiology, Mohammed I University/Mohammed VI University Hospital, Oujda, MAR
}

Corresponding author: Ismahane Lahmidi, lahmidiismahane@gmail.com

\begin{abstract}
Cardiac hydatidosis is an unusual disease and the interventricular septum is infrequently involved. It can cause various complications because of rupture and embolization. Interventricular septal cysts, may in rare conditions, induce symptoms corresponding to compression of the conduction pathway such as atrioventricular block. In this report, we present an uncommon case of cardiac echinococcus located in the basal part of the interventricular septum presenting as complete heart block managed by medical treatment and implantation of a permanent pacemaker.
\end{abstract}

Categories: Cardiology, Radiology, Infectious Disease

Keywords: cardiac hydatidosis, complete heart block, pace maker

\section{Introduction}

Hydatid disease, also known as hydatidosis, is engendered by infection with Echinococcus granulosus; it results in the constitution of one or more hydatid cysts situated frequently in the liver and lungs, and rarely in the kidneys, bones, spleen, muscles, and central nervous system. Cardiac involvement is very rare and it is encountered in only $0.5 \%$ to $2 \%$ of patients with hydatid disease $[1,2]$. Different segments of the heart can be affected; the location of a hydatid cyst in the interventricular septum is just $4 \%$ of cardiac cases $[1,2]$. Cyst growth is slow, only $10 \%$ of cardiac hydatid cyst patients are symptomatic [1]. Because of the lack of specific symptoms, the diagnosis of cardiac hydatid cyst can be challenging. We report a case of isolated cardiac hydatid cyst causing complete atrioventricular block which is infrequently observed in clinical presentation.

\section{Case Presentation}

A 67-year-old male patient was admitted to our hospital with chief complaints of asthenia and dyspnea. His past medical history was unremarkable. In his physical examination, blood pressure was recorded at 110/70 $\mathrm{mmHg}$ and his heart rate was $40 \mathrm{bpm}$. A complete atrioventricular block was revealed by an electrocardiogram (ECG) (Figure 1). Routine laboratory test results were normal. Transthoracic echocardiography showed a cystic formation of size $3.7 \times 2.5 \mathrm{~cm}$ situated in the basal part of the interventricular septum (Figure 2). We noted also a reduced left ventricular ejection fraction (40\%). Cardiac computed tomographic examination showed a well-defined cystic lesion in the interventricular septum,

Review began 11/25/2020 Review ended 12/02/2020 Published 12/07/2020

\section{Copyright 2020}

Lahmidi et al. This is an open access article distributed under the terms of the Creative Commons Attribution License CC-BY 4.0., which permits unrestricted use, distribution, and reproduction in any medium, provided the original author and source are credited. suggestive of hydatid cyst (Figure 3). Serologic tests for echinococcal infection were strongly positive. Thus, cardiac hydatid cyst with intraseptal involvement diagnosis was made.

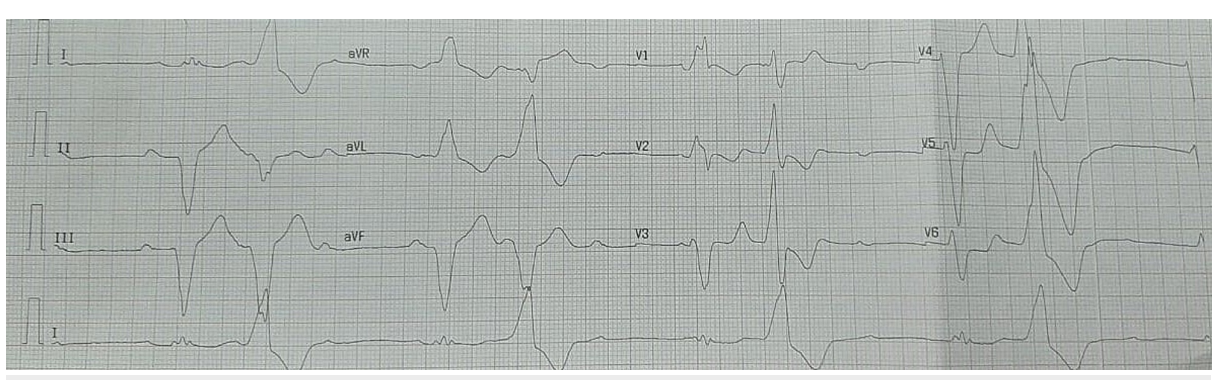

FIGURE 1: Electrocardiogram showing a complete atrioventricular block 


\section{Cureus}

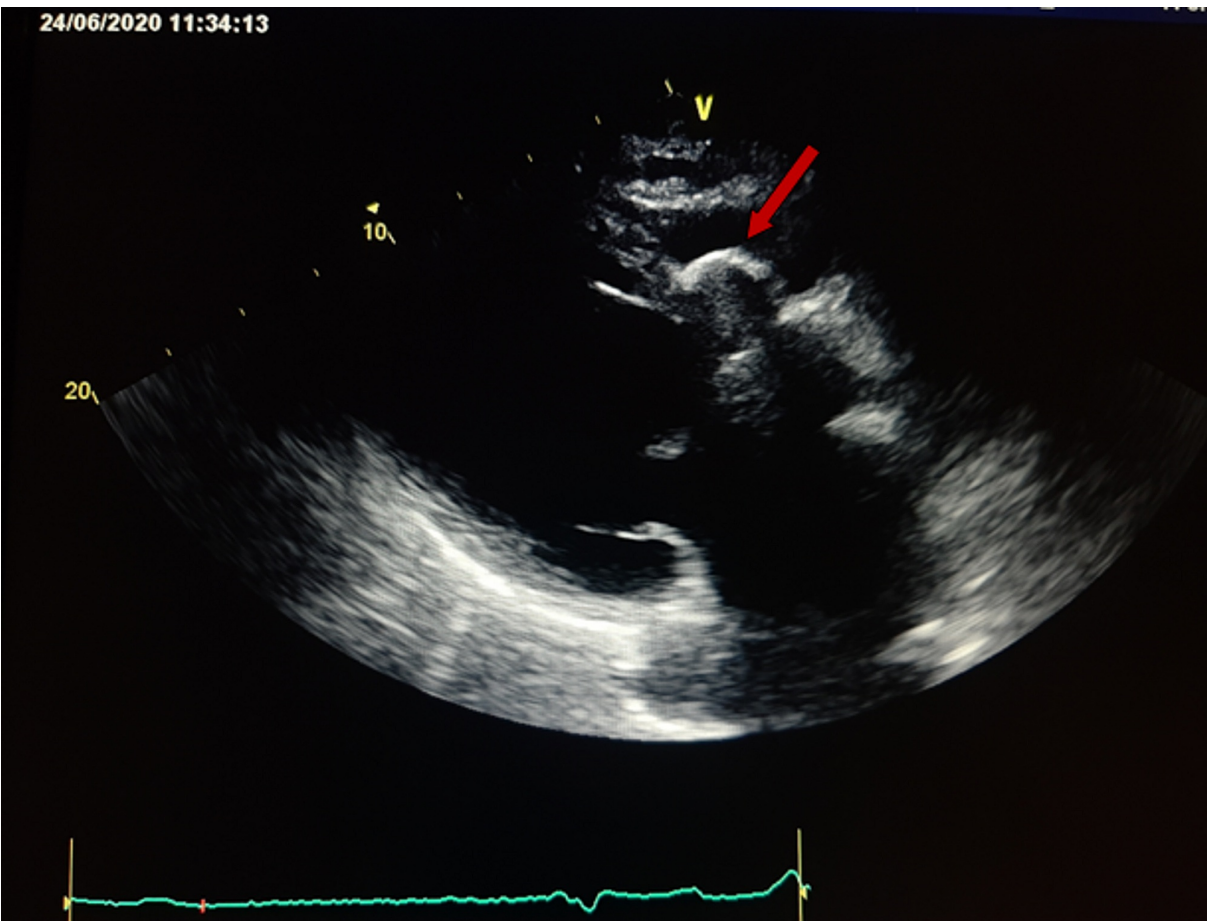

FIGURE 2: Transthoracic echocardiography showing cyst in the basal part of interventricular septum in the parasternal long-axis view

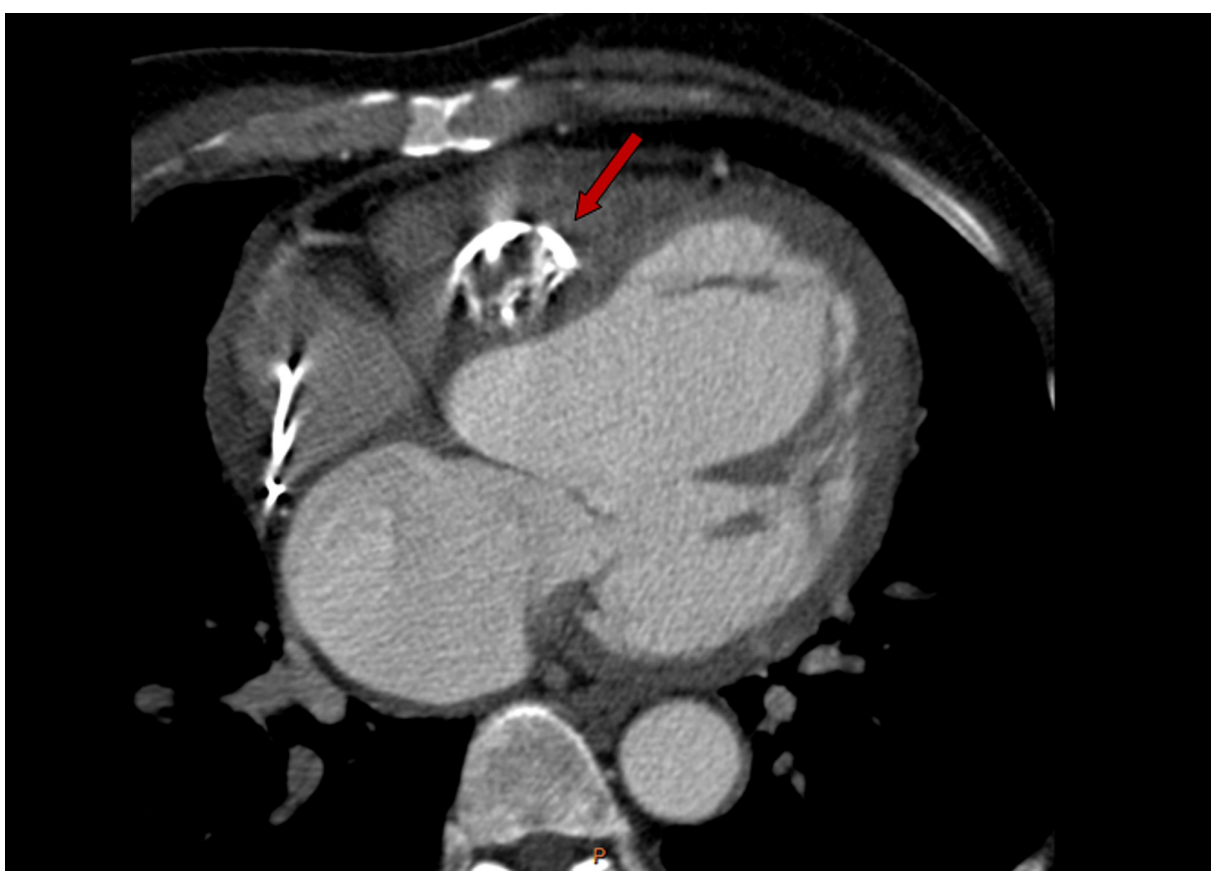

FIGURE 3: Computed tomography imaging showing a cyst located in basal interventricular septum with multiple calcifications

Owing to the size and location of the calcified cyst, surgical resection was not considered in order to minimize the risk of rupture of the contents; medical treatment with albendazole was initiated. A permanent pacemaker was implanted. The patient was discharged with albendazole treatment and was doing well at four months follow up.

\section{Discussion}


Human hydatidosis is an infection generated by the larvae of Echinococcus granulosus that remains endemic in Morocco. Primary hosts are dogs and other carnivores; some of the herbivorous and omnivorous animals are intermediate hosts, while humans are accidental hosts. Frequently affected sites by hydatid cysts are the liver and lungs, however, any body organ may be at risk for concern. Cardiac hydatid cysts are an uncommon condition, with a rate ranging from $0.5 \%$ to $2 \%$. The most involved part of the heart is the left ventricular owing to its maximum blood supply (60\%), followed by the right ventricle (10\%), pericardium (7\%), left atrium (6\%-8\%), right atrium (3\%-4\%), and interventricular septum (4\%) [1,2]. Williams reported the first case of cardiac localization in 1936 [3]. The clinical presentation ranges from asymptomatic to sudden death. Cardinal symptoms are chest pain, dyspnea, palpitations, and cough, they differ in accordance with the number, size, and site of the cysts [4]. As was described in our case, a cyst located in the basal septum close to the conduction system can induce atrioventricular block by compression of the conduction pathway.

Echocardiography is a performant and sensitive modality of choice to diagnose cardiac hydatid cyst. It allows a detailed characterization of the cysts, their size, number, localization, and their effect, and furthermore, any eventual complications. Computed tomography and MRI are essential for establishing the diagnosis, they furnish a detailed assessment of the lesions and their relationship with other structures [5]. In our case, the diagnosis was made by transthoracic echocardiography and confirmed by computed tomography assessment.

Standardized therapeutic management cannot be practicable owing to the diversified character of cardiac hydatidosis, however, surgery is still the treatment of choice for cardiac hydatid cysts, as well as in asymptomatic cases, to prevent life-threatening complications [6]. In some circumstances, medical treatment may be the only alternative, it could be indicated for small or calcified cysts, in elderly patients, or when surgery is contraindicated or refused by the patient [7]. In our case, because of the size, the calcified character of the cyst and increased operative risks, antihelminthic treatment was preferred and a permanent pacemaker was implanted.

\section{Conclusions}

To sum up, clinical presentations of cardiac hydatid disease are nonspecific and might mimic diverse cardiac diseases according to the location of the cyst. Cardiac hydatidosis should be considered in patients who present from areas where the disease is endemic.

\section{Additional Information \\ Disclosures}

Human subjects: Consent was obtained by all participants in this study. Conflicts of interest: In compliance with the ICMJE uniform disclosure form, all authors declare the following: Payment/services info: All authors have declared that no financial support was received from any organization for the submitted work. Financial relationships: All authors have declared that they have no financial relationships at present or within the previous three years with any organizations that might have an interest in the submitted work. Other relationships: All authors have declared that there are no other relationships or activities that could appear to have influenced the submitted work.

\section{References}

1. Shojaei E, Yassin Z, Rezahosseini O: Cardiac hydatid cyst: a case report. Iran J Public Health. 2016, 45:150710.

2. Yaman ND, Sirlak M: Cardiac hydatid cysts-review of recent literature . J Vet Med Res. 2017, 4:1102.

3. Murphy TE, Kean BH, Venturini A, Lillehei CW: Echinococcus cyst of the left ventricle. Report of a case with review of the pertinent literature. J Thorac Cardiovasc Surg. 1971, 61:443-50.

4. Fennira S, Kamoun S, Besbes B, et al.: Cardiac hydatid cyst in the interventricular septum: a literature review. Int J Infect Dis. 2019, 88:120-6. 10.1016/j.ijid.2019.09.004

5. Canpolat U, Yorgun H, Sunman H, Aytemir K: Cardiac hydatid cyst mimicking left ventricular aneurysm and diagnosed by magnetic resonance imaging. Turk Kardiyol Dern Ars. 2011, 39:47-51.

6. Brunetti E, Kern P, Vuitton DA: Expert consensus for the diagnosis and treatment of cystic and alveolar echinococcosis in humans. Acta Trop. 2010, 114:1-16. 10.1016/j.actatropica.2009.11.001

7. Tuncer E, Tas SG, Mataraci I, Tuncer A, Donmez AA, Aksut M, Yakut C: Surgical treatment of cardiac hydatid disease in 13 patients. Tex Heart Inst J. 2010, 37:189-93. 